\title{
Designing Proper Dishes for Quadriplegic Disables
}

\author{
Fatemeh Ghavam ${ }^{1}$, Ahmad Ali Noorbala ${ }^{2, *}$, Mohsen Rahimnia $^{2}$ \\ ${ }^{1}$ Department of Architecture, Young Researchers and Elite Club, Damavand Branch, Islamic Azad University, Damavand, IR Iran \\ 2 Department of Psychosomatic, Imam Khomeini Hospital, Tehran University of Medical Sciences, Tehran, IR Iran \\ *Corresponding author: Ahmad Ali Noorbala, Department of Psychosomatic, Imam Khomeini Hospital, Tehran, IR Iran. Tel.: +98-2161192421, Fax: +98-2166930330, E-mail: noorbala@ \\ tums.ac.ir.
}

Received: May 27, 2013; Revised: August 24, 2013; Accepted: October 6, 2013

\begin{abstract}
Background: This article ponders about one of the challenges faced by quadriplegic disables and investigates their capability in doing daily activities. Considering the high frequency of disability in the world, it is of vital importance to do research about this group of people many of who played significant roles in Iran-Iraq's war, serving them the right to be independent for handling their daily and personal activities. Any attempt to improve their perceptions of having control over their life and expand their choices for a preferred living situation has been proved to lead to a better quality of life in these people. Devices such as wheelchairs and walkers were traditionally devised to facilitate life for disables but this article took a further step to design special dishes compatible with the needs of spinal cord injury disables particularly quadriplegics. This measure is unprecedented in Iran and any comprehensive measure was not previously taken in the world.

Objectives: Facilitating eating habits of quadriplegics was the main goal of this project; designing dishes for quadriplegic disables.

Materials and Methods: In this study, field research was conducted, using a questionnaire, library, Internet, and an interview with quadriplegics and those who are incapacitated in using their hand.

Results: The designed dishes were given to several quadriplegic disables, which drew their attention pleasantly and satisfied them. The "Disables and Veterans Institute" also approved the dishes.

Conclusions: By using these dishes in all rehabilitation centers, pain and suffering of these patients can be alleviated and their independency can be improved. Although the "Disables and Veterans' Institute" welcomed the project, due to various reasons, this is yet to be massively produced.
\end{abstract}

Keywords: Quadriplegia; Rehabilitation; Cooking and Eating Utensils

\section{Background}

Spinal cord injury (SCI) is a devastating condition often affecting young and healthy individuals around the world. This debilitating condition not only creates enormous physical and emotional cost to individuals but also is a significant financial burden to the society (1). Spinal cord injury occurs in various countries throughout the world with an annual incidence of 15 to 40 cases per million, with the causes of these injuries ranging from motor vehicle accidents and community violence to recreational activities and workplace-related injuries. Survival has improved along with a greater appreciation of patterns of presentation, survival, and complications. Despite much work having been done, the only treatment to date known to ameliorate neurologic dysfunction that occurs at or below the level of neurologic injury has been intravenous methylprednisolone therapy. Much research over the past 30 to 40 years has focused on elucidating the mechanisms of spinal cord injury, with the complex pathophysiologic processes slowly being unraveled. With a greater understanding of both primary and secondary mechanisms of injury, the roles of calcium, free radicals, sodium, excitatory amino acids, vascular mediators, and apoptosis have been elucidated (2). A systematic review by Rahimi Movaghar and colleagues found the incidence of SCI in developing countries to be $25.5 /$ million/year (95\% CI: 21.7 - 29.4/million/year) ranging from 2.1 to 130.7 | million/year. Males comprised 82.8\% (95\% CI: 80.3 - 85.2) of all SCIs with a mean age of 32.4 years (95\% CI: 29.7-35.2). The two leading causes of SCI were found to be motor vehicle crashes (41.4\%; 95\% CI: 35.4 - 47.4) and falls (34.9\%; 95\% CI: 26.7 - 43.1). Complete SCIs were found to be more common than incomplete injuries (complete SCI: $56.5 \%$;

Implication for health policy/practice/research/medical education:

This article ponders about one of the challenges faced by quadriplegic disables and investigates their capability in doing daily activities. Any attempt to improve their perceptions of having control over their life and expand their choices for a preferred living situation has been proved to lead to better quality of life for these people. Devices such as wheelchairs and walkers were traditionally devised this article took a further step to design special dishes compatible with the needs of spinal cord injury disables particularly quadriplegics. This measure is unprecedented in Iran and any comprehensive measure was not previously taken in the world.

Copyright (C) 2014, Tehran University of Medical Sciences. This is an open-access article distributed under the terms of the Creative Commons Attribution License, which permits unrestricted use, distribution, and reproduction in any medium, provided the original work is properly cited. 
95\% CI: 47.6-65.3; incomplete SCI: 43.0\%; 95\% CI: 34.1-52.0). Similarly, paraplegia was found to be more common than tetraplegia (paraplegia: 58.7\%; 95\% CI: 51.5 - 66.0; tetraplegia: 40.6\%; 95\% CI: 33.3 - 48.0) (3). Prevalence of traumatic spinal cord injury is estimated to be around 440 per million in the population of Tehran (4). Whiteneck G. and colleagues investigated environmental barriers reported by people with spinal cord injury (SCI)(5). The top five environmental barriers reported by subjects with SCI, in descending order of importance, were the natural environment, transportation, need for help at home, availability of health care, and governmental policies. A review by Hammell (6), concerning quality of life following high spinal cord injury explored relevant articles for the period of 1990 - 2003 and tried to figure out the main contributing factors to a higher quality of life among affected individuals. Here are some of findings:

-A small number of researchers have reported that perceptions of satisfactory QOL are negatively correlated with the level of spinal cord lesion, although this association is not generally found to be statistically significant. In contrast, a substantial body of research has found no correlation between QOL/life satisfaction and level of SCI: the degree of impairment or extent of physical independence.

-Quantitative research among people with all levels of SCI has demonstrated a positive correlations between QOL/life satisfaction and self-assessed health, perceived social support, social functioning/integration, mobility, accessibility (home/health care), preferred living situation, adequate income, perceptions of having control over one's life, marital status, satisfaction with relationships,community access/participation and satisfaction with occupational engagement and/or employment status.

- Life satisfaction/QOL has been reported to be negatively correlated with re-hospitalizations/perceptions of poor health (including respiratory problems and pressure sores), pain, spasticity, inadequate income, reduced life opportunities, reduced social interaction, loneliness and boredom, unemployment/dissatisfaction with occupations, reduced mobility, residence in a nursing home, and perceptions of having reduced control over one's life.

- The researcher suggested a solution for the problems associated with SCI at both the cellular and the societal levels. Considering the fact that social, physical, economic, legal and political environments increase the problems confronted by people with SCI and reduce their quality of life, interventions must be targeted not solely at individuals, teaching them to live in a world designed to meet the needs of the dominant population, but also at public policies and community planning. Commitment to reduce, for example, pain, spasticity, pressure sores and respiratory problems (factors equated with poor QOL) should be matched by a commitment to reduce, for example, nursing home admissions, occupa- tional deprivation, inadequate access to health-care and poverty (also factors associated with poor QOL). How this might be accomplished would be a worthy avenue for future research.

Safilios-Rothschild (7) found that 'rehabilitation entails a highly stressful re-socialization process into an 'inferior' status' and that the process of 'accepting' disability necessitates 'the relinquishing of majority status rights'. 'Adjustment' to disability, as argued, is not so much a psychological process but a process of adjusting to changes in others' perceptions, in social positioning, and in access to educational, economic and social opportunities. Indeed, the onset of impairment has been described as the experience of a 'fall from privilege'. In 2011 Babamohamadi and colleagues studied coping strategies in eighteen Iranian patients with SCI and found the most commonly used strategies were seeking help from religious beliefs (understanding the disease as a divine fate and as a spiritual combat), hope and making efforts towards independence/selfcare, emphasizing the importance of independence in this group (8). The same research team in another published research assessed barriers to and facilitators of coping with spinal cord injury for Iranian patients (9). After using purposeful sampling for selection of the participants, semi-structured interviews were held for data collection. Lack of "knowledge", "financial resources", "employment opportunities", "suitable facilities and accessibility" as well as "societal acceptance and support" emerged as barriers; and having "self-confidence", "religious beliefs", "support networks" and "positive thinking" emerged as facilitators in coping with spinal cord injury. Participants believed that with these hindering factors in place, adapting to their new condition had been difficult, therefore they could only manage to adapt partially to their situation. Since disabled people of the society are the target group for the present article, different sorts of disabilities are discussed briefly. Disabilities can be divided into five groups: 1) tetraplegia (quadriplegia), 2) paraplegia, 3) spinal cord injury (SCI), 4) cerebral palsy (CP), 5) multiple sclerosis (MS). Tetraplegia is caused by extensive damage to the brain or the spinal cord. The injury, which is known as a lesion, causes victims to lose partial or total function of all four limbs, meaning the arms and legs and the torso. Typical causes of this damage are trauma. However, when the paralysis affects the lower portion of the spinal cord, the quadriplegics have more control over their organs. Unfortunately, devices and tools specific for disables have been rarely devised in Iran. Therefore, this project aims at designing special dishes for this group of people.

\subsection{Classification Based on Level of Lesion}

Depending on the specific location of the spinal cord injury, severity of lesion varies. Generally, the nearer the damage to the neck, the more severe lesion will result and more organs will be paralyzed. Classification includes: 
1- Quadriplegia:

a) Injuries at the C-1/C-2 part may cause "high-level quadriplegia".

b) Injuries at the C-6/T1 portion lead to "low-level quadriplegia" (target group of the present project).

2-Paraplegia:

a) Injuries at the $\mathrm{T} 2 / \mathrm{T} 5$ portion generate "high-level paraplegia".

b) Injuries at the T6/T12 portion are the sources of "middle-level paraplegia".

c) Injuries at the L-1/L-5 portion lead to "low-level paraplegia”.

\subsection{A Brief Look Over Spinal Cord Injuries}

1- Injuries at the $\mathrm{C}-1 / \mathrm{C}-2$ portion result in the loss of function of the diaphragm

2-Injuries at the C-3/C-4 portion typically result in loss of diaphragm function and biceps.

3- Injuries at $\mathrm{C}_{5}$ portion results in potential loss of function at biceps, and complete loss of function at the wrists and hands.

4- Limited wrist control and complete loss of elbow extensor function but well-functioning biceps indicate injury at the $\mathrm{C} 6$ portion.

5-If wrist extensors and internal hand muscles work well but elbow extensors weaken, injury is at the $\mathrm{C} 7$ portion.

6- Intact elbow extensors and weak internal hand muscles indicate injury at the C8 portion.

7- Injury at T1 or lower parts doesn't affect hand function.

8- Injury at L1 or higher parts leads to complete loss of function of thigh adductors.

9- Injury at L2 leads to weak thigh adductors and complete loss of function of knee extensors.

10- Intact thigh adductors, weak knee extensors and paralyzed tibialis anterior muscle indicate injury at the L3 level.

11- Intact knee extensors and weak tibialis anterior muscle and paralyzed big toe extensor indicateL4 injury.

12- If tibialis anterior muscle is intact, thumb extensor acts weakly, and biceps muscle lose function, injury is at the L5 level.

13- If big toe extensor works well, biceps muscles are weak and anus sphincter stop working, injury is at S1 level.

14- Injury at S2 level means that biceps muscles are intact whereas anus sphincter works poorly.

\section{Objectives}

With regard to the large number of quadriplegics in Iran and all over the world and considering the motto "equalization of opportunities", and the significance of fulfilling the needs of these people, this article seeks to design a special dish.

\section{Materials and Methods}

When available cases in Iran were investigated, it was detected that they didn't have access to any special dish and those who were unable to use their hand - called "mouth disabled"- used steel tea pots for drinking water. Hot or cold water was put next to their face and they had to drink through its pipe. For eating food, they were generally dependent on a nurse who served six people in a room so their food usually went cold. If they ate food by themselves, it would drop out of the dish. When interviewed, they expressed their dissatisfaction with their dishes and their materials. Porcelain dishes were not used because of their maintenance, cleaning, and fragility caused problems. On the other hand, they lacked shelves for keeping them and they were not washed well. Following field research and some questionnaires, it was concluded that disables prefer to eat themselves and they were interested in porcelain or ceramic or any materials except plastic and metallic dishes. The only problem in designing porcelain dishes is their fragility so they need special holders to stop them from slipping or they must be made of unbreakable materials such as Pyrex.

\section{Results}

Regarding investigations and damaged segments, it was concluded that C6-T1 quadriplegic disables face problems with opening and closing their wrist and arm. They can either eat independently but by aid of specific tools and devices or they need a nurse. The designed dishes (Figure 1) were analyzed in terms of material, shape, and form. They are deep enough so that food won't drop out and enable disables to eat tidily and independently (Figure 2). The designed cups are equipped with two holders which guarantee better gripping in order to prevent falling. These dishes can be applied both at home and in rehabilitation centers. If they are to be used at home, rubber placemat are designed which prevent slipping of dishes. If these dishes are to be used in rehabilitation centers, they need to be shatterproof so that if dropped, their noise would not disturb other patients. Unfortunately, at the moment, there are no shatterproof and Pyrex dish factories in Iran. That's why they can't be mass-produced.

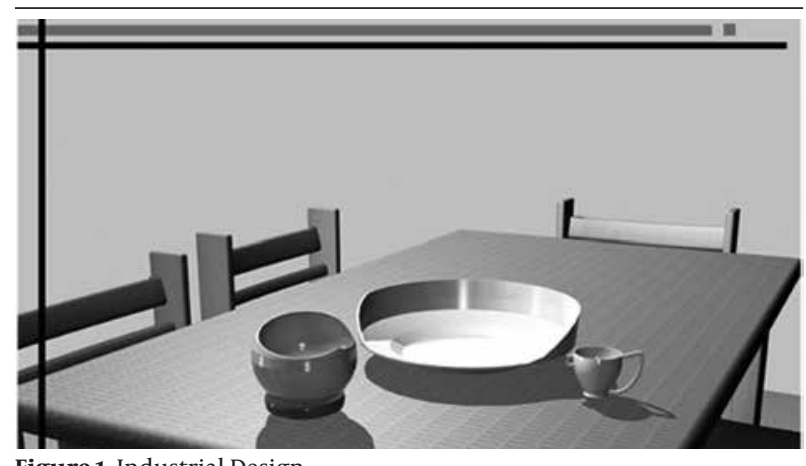

Figure 1. Industrial Design 


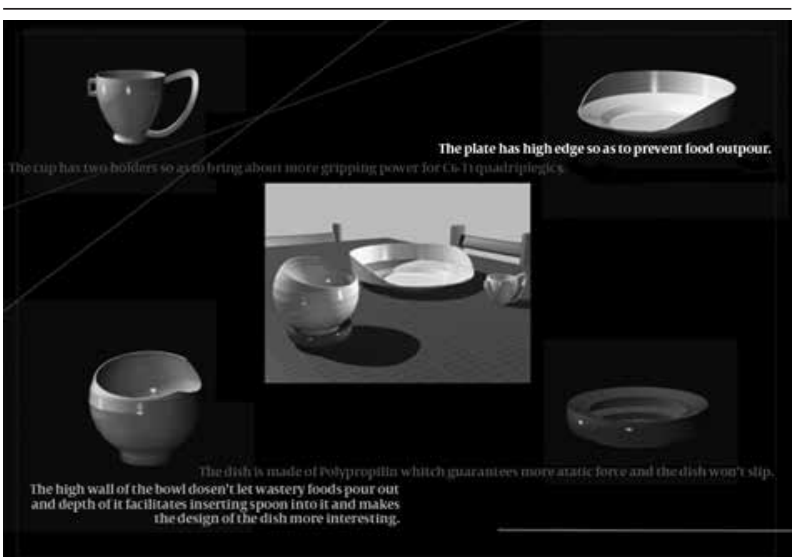

Figure 2. Design Justification

\section{Discussion}

It is a massive and important leap to design these kinds of dishes, which enable C6-T1 quadriplegic disables to eat independently using nice, clean and ergonomic dishes. It facilitates rehabilitation of these patients. These dishes are of great value as they satisfied the patients. It is expected that one day in the near future, patients from all over the world could utilize these dishes.

\section{Acknowledgements}

The Authors would like to thank the Foundation of Martyrs and Veterans affairs, especially Mr. Aalami who contributed to resolve the technical problems.

\section{Authors' Contribution}

Study concept and design: Fatemeh Ghavam; Manage- ment and supervision: Dr. Noorbala; Scientific background: Dr. Rahimnia.

\section{Financial Disclosure}

The Authors declare no financial disclosure.

\section{Funding/Support}

The research has not utilized any financial aid from any organization.

\section{References}

1. Ackery A, Tator C, Krassioukov A. A global perspective on spinal cord injury epidemiology. J Neurotrauma. 2004;21(10):1355-70.

2. Sekhon LH, Fehlings MG. Epidemiology, demographics, and pathophysiology of acute spinal cord injury. Spine (Phila Pa 1976). 2001;26(24 Suppl):S2-12.

3. Rahimi-Movaghar V, Sayyah MK, Akbari H, Khorramirouz R, Rasouli MR, Moradi-Lakeh M, et al. Epidemiology of traumatic spinal cord injury in developing countries: a systematic review. Neuroepidemiology. 2013;41(2):65-85

4. Rahimi-Movaghar V, Saadat S, Rasouli MR, Ganji S, Ghahramani M, Zarei MR, et al. Prevalence of spinal cord injury in Tehran Iran. J Spinal Cord Med. 2009;32(4):428-31.

5. Whiteneck G, Meade MA, Dijkers M, Tate DG, Bushnik T, Forchheimer MB. Environmental factors and their role in participation and life satisfaction after spinal cord injury. Arch Phys Med Rehabil. 2004;85(11):1793-803.

6. Hammell KW. Exploring quality of life following high spinal cord injury: a review and critique. Spinal cord. 2004;42(9):491-502.

7. Safilios-Rothschild C. Disabled persons' self-definitions and their implications for rehabilitation. In: Brechin AP, Glaister A, Liddiard P, Swain J, editors. Handicap in a Social World: A Reader. London: Hodder and Stoughton; 1981.

8. Babamohamadi H, Negarandeh R, Dehghan-Nayeri N. Coping strategies used by people with spinal cord injury: a qualitative study. Spinal Cord. 2011;49(7):832-7.

9. Babamohamadi H, Negarandeh R, Dehghan-Nayeri N. Barriers to and facilitators of coping with spinal cord injury for Iranian patients: a qualitative study. Nurs Health Sci. 2011;13(2):207-15. 